\title{
Using Mathematical and Statistical Model to Forecast the Path of Infection by Covid-19 in the Kingdom of Saudi Arabia
}

\author{
Tarek M. Omara ${ }^{a, b}$, Khaled A. Harby \\ ${ }^{\mathrm{a}}$ Department of Mathematics, Faculty of Science, Islamic University, Saudi Arabia \\ ${ }^{b}$ Department of Statistics, Mathematics and Insurance, Faculty of Commerce, Kafrelsheikh University, Egypt \\ 'Department of Linguistics, Faculty of Arabic Language, Islamic University, Saudi Arabia
}

\begin{abstract}
Saudi Arabia, like any other part of the earthly globe, has been exposed to the Covid-19 pandemic. The first case appeared on March 3, 2020, followed by an increase in the number of infections until it reached thousands with the numbers on the rise. Therefore, adopting clear strategies to deal with the pandemic according to specific data on its size is necessary. In this study, the time series of the number of infections and deaths were analyzed to study the behavior of the pandemic over time. The cumulative curve of the phenomenon was analyzed to show the extent of the pandemic's decline or spread. On the other hand, the time curve of the number of cases of the pandemic was fitted based on a set of mathematical and statistical models, which were divided into three sections [nonlinear growth model, Susceptible, Exposed, Infectious, Recovered (SEIR) model, regression model] to attain the best possible fitting of the relationship curve. The results show that the Weibull model and Polynomial model at $(n=4)$ are the best models for fitting the relationship at short run and the SEIR model gives better relationship fitting at long run. In conclusion, there is a tendency for the disease to decline during the short period, while expecting other waves of the epidemic that will recede in the long term with the emergence of a suitable vaccine.
\end{abstract}

KEYWORDS: Covid-19, Fit the curve, Nonlinear growth models, SEIR model, Regression model

\section{INTRODUCTION}

The world is currently witnessing the risk of infection with Corona Virus, Covid-19, which was defined by WHO as a pandemic in $2020^{1}$. This virus is among the group of seven types of coronaviruses that infect the respiratory system. Its severity ranges from mild cold symptoms to respiratory syndrome symptoms followed by acute lung inflammation to pulmonary failure that eventually leads to death. To humans, the most dangerous of the seven forms of the Coronavirus is SARS-CoV and MERS-CoV. ${ }^{2}$ It is conspicuous that this

\section{Corresponding Author:}

Dr. Tarek Mahmoud Omara

42351 Prince Nayef bin Abdulaziz,

Statistics and Information Center,

Islamic University, Medina, Saudi Arabia.

Tel No : +966546781595

E-mail : Tarek_em@yahoo.com virus has the ability to spread rapidly, doubling the severity as proven by the failure of health systems all over the world to deal with patients in a timely and optimal way, especially since a treatment is not yet available for the disease. A large number of infected people may not realize that they have the disease unless after a period of time, which makes them a typical means of spreading the disease before they are isolated from the rest of the healthy people. ${ }^{1}$ As a result, the total number of cases of infection reached 16 million and 380 thousand people on 5/9/2020 around the world, with the most cases reported in the United States of America with approximately 4 million and 365 thousand. Brazil comes in the second with about 2 million and 419 thousand cases, ${ }^{1}$ which led many countries to implement total or partial isolation to prevent the spread of the disease. In the Arab world, Saudi Arabia topped the number of infections as the 
total numbers summed up to 266 thousand and 941 on the same date.

With the increase in economic and social pressures and the flow of accurate information about the disease, the low growth rate in the number of deaths and the high rates of recovery, countries began to break the restrictions of isolation and returned to normal life with the presence of precautionary measures. The preliminary indicators of the spread of the disease show that it may continue for months until it reaches the stages of decline. Several studies that used mathematical models to predict numbers of infections, cures and deaths have been applied in many countries, including those using a set of mathematical models to fit the curve of disease growth. ${ }^{3-6}$ These models were built in the initial periods of the disease where the data available and medical knowledge of the disease were limited and therefore, the degrees of accuracy of these studies varied. Furthermore, considering the studies related to the pandemic in the Kingdom of Saudi Arabia, we found that there are modest contributions in this direction. Saleh et $\mathrm{al}^{7}$ conducted a study that predicts the infected population in the Kingdom of Saudi Arabia based on the Autoregressive-moving-average (ARMA) model. It is noted that, in addition to the temporal trend of the development of the spread of the disease, there is a need to study the effect of other factors that may contribute to its spread such as the effect of lockdown, environmental and social factors. By studying the spread of the disease, Villalobos ${ }^{8}$ suggested using generalized extrapolation models to predict the growth rate of the disease in the short term due to the poor information related to the disease. In fact, with the increase in the time period during which the disease has spread, more data about it have been obtained and thus accuracy in predicting the spread. On the other hand, some studies, such as Christine $^{6}$ used the Susceptible, Exposed, Infectious, Recovered (SEIR) model to fit the infection growth curve. This method is considered suitable for predicting the number of infections in the long run.

It has been observed that there are many mathematical and statistical models that can be used to describe the behavior of the disease in the future. The patterns of these models differ in terms of degree of complexity and may require a large amount of data, which may be unavailable, especially in the light of newly emerging pandemic. Therefore, these models must take into account making a balance between the lack of data and the urgent need to conceive about future disease behavior and how to deal with it. In addition, many models can only develop a short-term perception of disease outbreaks such as time series models, which are based on case data only. The current study provides a wide range of mathematical and statistical models to follow the growth curve of Covid-19 disease in the Kingdom of Saudi Arabia. Additionally, with regards to mathematical models, we are going to make use of the SEIR model and nonlinear growth models, but with respect to statistical models, the study will use the regression model at different forms. We aimed to determine the best model that can be used to forecast the infection at short and long terms, and then compare predictions using this model enabling us to decide the best way to deal with it.

\section{MATERIALS AND METHODS}

We reviewed a set of mathematical and statistical models that can be used to predict the temporal development of the spread of pandemics. Owing to the fact that the growth curve of the Covid-19 virus is close to S-shape, it is expected that the logistic model and its special cases, the SEIR model, as well as the quadratic and polynomial regressions are the closer and probable models that can be relied upon to fit the relationship curve.

\section{Nonlinear growth models}

In many cases, linearity is unable to accurately describe the behavior of the growth of different phenomena over time. As a result, a large group of nonlinear growth models have emerged that can be relied upon to fit the growth curve to phenomena with nonlinear characteristics. In case of infection, the behavior often takes nonlinear way. Therefore, the current study used the nonlinear models like logistic and generalized logistic, Weibull, Mitscherlich, and Gompertz models to fit the Covid-19 pandemic curve. 
The logistic model is based on the logistical growth function, which describes a high growth state at the beginning of the spread of the phenomenon and then returns to decline at the end, and thus takes the form of the shape $\mathrm{S}$. This function has a maximum extent that the phenomenon can reach, and this is not found in the exponential growth function. However, the logarithmic growth function can be represented in the form below:

$$
\frac{d f(t)}{d t}=r_{\max } f(t)\left(\frac{k-f(t)}{k}\right) \max _{t \rightarrow \infty} f(t)=k
$$

Here, $f(t)$ is a function of the predicted phenomenon variable at time $t, k$ the maximum capacity and $r_{\max }$ maximum rate of variability of the phenomenon.

The equation in (1) can be represented with flexibility in the following form:

$$
f\left(t ; \beta, k, r_{\max }\right)=\frac{k}{1+\beta \mathrm{e}^{-r_{\max }}}, \beta=\frac{k}{y_{0}}-1
$$

In this equation, ${ }^{y_{0}}$ is the value of $\mathrm{y}$ at the beginning of the phenomenon. Richard 9 has proposed developed forms of a generalized logistic model called Richards' curve. This model gives a higher degree of flexibility in S -shaped curves and it takes the form below:

$$
f\left(t ; \beta, m, k, r_{\max }\right)=\frac{k}{\left(1+\beta_{\mathrm{e}}-r_{\max }\right)^{\frac{1}{m}}}, \beta=\frac{k}{y_{0}}-1
$$

Hitherto, positive scale parameter $m$ has an effect near the maximum limit of the curve.

\section{Weibull model}

Fréchet ${ }^{10}$ suggested a Weibull model that takes the following form:

$$
f\left(t ; \beta, m, r_{\max }\right)=r_{\max } t^{m-1} \beta e^{-\left(r_{\max } t\right)^{m}}
$$

In this equation, an exponential model can be obtained when we set $\mathrm{N}=1$

\section{Mitscherlich model}

Mitscherlich model was one of the first models used in the estimation of the growth function by Mitscherlich in 1909 and it takes the following form:

$$
f\left(t ; \beta, k, r_{\max }\right)=k-\beta e^{-r_{\max } t}
$$

\section{Gompertz model}

The Gompertz model is a special case of the logistical model and takes the form below:

$$
f(t ; \beta, k)=k e^{-\beta e^{-r_{\max } t}}
$$

To choose the best model from the previous models, which has the best goodness of fit and can be relied upon for prediction, we are going to use the coefficient of determination $\left({ }^{2}\right)$ that can be derived through the relationship:

$$
R^{2}=\frac{S S R}{S S T}=1-\frac{S S E}{S S T}
$$

where SSR is sum square regression and SST is sum square total.

\section{Susceptible-Infected-Recovered (SIR) model}

The SIR model is considered as one of the basic simulation models that are used to predict trends of the spread of pandemics over time in the presence of a large population. Kermack and McKendrick, ${ }^{11}$ have suggested it to estimate the extent of the spread of plague and cholera. In fact, this model gives an idea about the extent of disease spread and when it becomes a pandemic along with the extent of its recession and whether cases will remain after the disease recedes or not. This model also gives results that are more accurate as the time period of the disease increases. In reality, this model is good for dealing with pandemics when it involves a large number of people. ${ }^{12}$ 
Moreover, this model consists of three main parts: 'S' expresses the number of individuals exposed to the disease, ' $\mathrm{I}$ ' is the number of sick individuals and carriers of the disease, and ' $\mathrm{R}$ ' is the number of individuals who were excluded due to death or because they became immune to the disease by recovery. This model is dynamic as it allows individuals to move through these three parts over time. The SIR model is built according to the following equations:

$$
\begin{aligned}
& \frac{d S}{d t}=-\frac{\beta I S}{N} \\
& \frac{d I}{d t}=\frac{\beta I S}{N}-\gamma \\
& \frac{d R}{d t}=\gamma I
\end{aligned}
$$

where ${ }^{\beta}$ is the average number of infected individuals as a result of mixing with one person during a unit of time, $r$ is the recovery rate and $\mathrm{N}=\mathrm{S}+\mathrm{I}+\mathrm{R}$ is the population.

In SIR model, the first equation represents the stage of transition of the disease from susceptible to the stage of infection and the third equation represents the stage of transition from infection to the stage of recovery. Two key indicators can be extracted from the SIR model, which are the control reproduction number $\left(R_{0}=\frac{\beta}{\gamma}\right)$ and transition rate $\left(F=\beta \frac{I}{N}\right)$.

\section{SEIR model}

In many pandemics, there may be an incubation stage for the disease and the individual may be a carrier of the infection or suffers from the disease, but the symptoms of the disease do not appear. Therefore, Aron ${ }^{13}$ added a new element to the components of the SIR model, which is Exposed (E) for the new model to become SEIR consisting of four parts (Susceptible-ExposedInfective- Recovered). There is an indicator in this model, and it is $R_{0}$, which expresses the infection of the disease or the number of people that can be infected by one infected person over a period of time. This variable is considered as an indicator of the extent of the decline or spread of the disease during a specific period of time, and it can be built according to the following equations:

$$
\begin{aligned}
& \frac{d S}{d t}=\mu_{B} N-\mu_{D} S-\beta \frac{I}{N} S \\
& \frac{d E}{d t}=\beta \frac{I}{N} S-\left(\mu_{D}+a\right) E \\
& \frac{d I}{d t}=a E-\left(\mu_{D}+\gamma\right) \\
& \frac{d R}{d t}=\gamma I+\vartheta R
\end{aligned}
$$

Here $\mathrm{a}$ is the incubation rate, $\vartheta^{\vartheta}$ is the rate at which recovered cases turn susceptible, ${ }^{\mu_{B}}$ is the birth rate and $\mu_{D}$ is the death rate. Gaurav ${ }^{5}$ modified the SEIR model so that the numbers of births, deaths and recovered cases are excluded, thus they will not be vulnerable to infection again. So the model appears in the form below:

$$
\begin{aligned}
& \frac{d S}{d t}=-\beta \frac{I}{N} S+\vartheta R \\
& \frac{d E}{d t}=-\beta \frac{I}{N} S-a E \\
& \frac{d I}{d t}=a E-\gamma,
\end{aligned}
$$

$$
\frac{d R}{d t}=\gamma I-\vartheta R
$$

\section{Regression models}

The regression model is considered one of the statistical models that can be used to demonstrate the effect of independent variables on a dependent variable, and it can also be used to predict the behavior of the dependent variable in the future. There are different forms of regression models, including simple regression and multiple regression, which can be linear or nonlinear.

Below is the general formula for a linear regression model:

$$
Y=X \beta+\epsilon
$$


Here, ${ }^{Y}$ vector of dependent variables with $\mathrm{n}$ is value of observation, ${ }^{\mathrm{X}}$ is explanatory matrix, ${ }^{\beta}$ is vector of coefficients and $E^{E}$ is vector of error term.

There are special cases of regression models, including:

$$
\begin{aligned}
& Y=\beta X^{2}+\epsilon \quad \text { (Quadratic form) } \\
& Y=\beta_{0}+\beta_{1} X+\beta_{2} X^{2}+\cdots+\beta_{2} X^{n}+\epsilon
\end{aligned}
$$

(Polynomial form)

To show the goodness of fit of previous regression models one can rely on $\mathrm{R}^{2}$.

In the current study, we used the time as an explanatory variable, number of infections as a dependent variable and the coefficients refer to the effect of change of time by one unit to the infection.

\section{Data analysis}

In order to access the results of the application of the proposed mathematical and statistical models to predict future infections with the Coronavirus, data related to this virus in the Kingdom of Saudi Arabia were collected through Worldometer, ${ }^{14}$ which gives daily data on Covid -19 cases in a large group of countries. In fact, the data showed that the first case of the Coved-19 virus was reported on March 3, 2020. Following this, the study relied on a daily time series until September 5, 2020. Figure (1) shows the development of cases during this period and similarly, Figure (2) and Figure (3) show the cumulative development of Covid-19 infection and death during the same period.

From Figure (1), we noticed that the spread of the disease took an accelerating trend from March to June 2020 , and a decline was seen during the month of July 2020.

Moreover, we have found find that there was a noticeable decrease from May 21 to May 30, 2020 during which the number of cases fell from 2593 to 1618 due to the precautionary measures adopted by the Saudi government during the Eid al-Fitr period, which included a complete lockdown and curfew implemented
24 hours a day. However, this was followed by a sharp increase in the number of infections until it reached its peak of 4919 cases on June 18, 2020. In the same vein, we took notice of the movement of cases towards gradual decrease until September 5, 2020, when it reached 2201. This is an indication of the high degree of social awareness and the application of strict precautionary measures by the competent authorities and society as well. When we considered the infection curve, we found that it took a shape close to the logistic curve and the quadratic function curve noting a trend of tilt to the left, pointing the movement of the disease towards recession unless we otherwise witnessed a new wave of the disease during the fall and winter seasons.

Figure 1: The number of daily infections caused by Covid-19 in the Kingdom of Saudi Arabia from March 3 to September 5, 2020

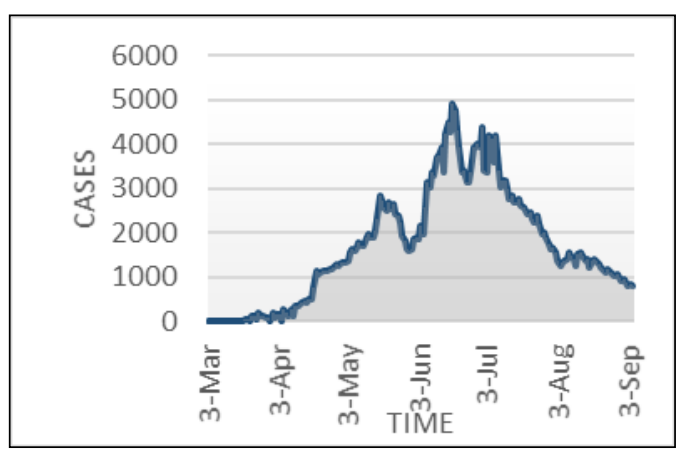

\section{RESULTS}

In this section, we reviewed the results that were attained through the application of the proposed mathematical and statistical models to study the behavior of the Covid-19 pandemic in the future. We used the IBM SPSS statistics 20 packages and MATLAB to analyze the data and draw conclusions.

\section{The results of estimating the nonlinear growth models}

The results of estimating the parameters of nonlinear growth models have been summarized in Table (I) to arrive at the best model to fit the curve of the Covid-19 infection in the Kingdom of Saudi Arabia. This table contains the value of the models' parameters and the value of $R^{2}$ which represents the statistical measure of the quality of the fitting of the relationship (the goodness of fit). 
Table I: The parameter and coefficient of determination the nonlinear growth models

\begin{tabular}{|c|c|c|c|c|c|c|}
\hline \multicolumn{7}{|c|}{ Logistic model } \\
\hline Parameter & $\mathbf{k}$ & $\beta$ & $\mathbf{r}_{\max }$ & $\mathbf{n}$ & $\mathrm{m}$ & $R^{2}$ \\
\hline Value & 3321.59 & 89.02 & 0.071 & 184 & --- & 0.558 \\
\hline \multicolumn{7}{|c|}{ Generalized logistic model } \\
\hline Parameter & $\mathbf{k}$ & $\beta$ & $r_{\max }$ & $\mathrm{n}$ & $\mathrm{m}$ & $R^{2}$ \\
\hline Value & 3320.3 & 99.78 & 0.072 & 184 & 1.039 & 0.862 \\
\hline \multicolumn{7}{|c|}{ Weibull model } \\
\hline Parameter & $\mathrm{k}$ & $\beta$ & $r_{\max }$ & n & $\mathrm{m}$ & $R^{2}$ \\
\hline Value & -- & 2.707 & 0.008 & 184 & 3.702 & 0.906 \\
\hline \multicolumn{7}{|c|}{ Mitscherlich model } \\
\hline Parameter & $\mathrm{k}$ & $\beta$ & $r_{\max }$ & n & $\mathrm{m}$ & $R^{2}$ \\
\hline Value & 5655.53 & 6423.54 & 0.008 & 184 & --- & 0.452 \\
\hline \multicolumn{7}{|c|}{ Gompertz model } \\
\hline Parameter & $\mathrm{k}$ & $\beta$ & $r_{\operatorname{mix}}$ & n & $\mathrm{m}$ & $R^{2}$ \\
\hline Value & 3381.52 & 13.789 & 0.049 & 184 & --- & 0.568 \\
\hline
\end{tabular}

From Table (I), we notice that the best model to fit the curve of infection with Covid-19 disease in the Kingdom of Saudi Arabia is the Weibull model, where $R^{2}$ reached 0.906. The Generalized logistic model comes in second position in terms of the best models to fit the curve where ${ }^{2}$ reached 0.862 , noting that all models are valid for fitting the relationship as the $R^{2}$ value ranged between 0.79 and 0.906 and all are significant at $\alpha=0.05$.

\section{The results of estimating the SEIR model}

In order to estimate the SEIR model, the SEIR algorithm proposed by Gaurav ${ }^{5}$ was used. Thus, given the incubation period of 14 days according to the World Health Organization, ${ }^{1} a_{0}=\frac{1}{D}=\frac{1}{14}$ where D is the infection period and if we assume that each affected individual will cause an infectious transmission every three days, $\beta_{0}=1 / 3$. According to the initial phenomena of the disease, Xia et $\mathrm{a}^{15}$ believes that the average recovery period is 12.9 days, which was also considered in this study. Therefore, the recovery rate is $\gamma_{0}=\frac{1}{R}=\frac{1}{12.9}$. It was assumed that $\vartheta_{0}=0.002$, and $\mathrm{N}$ represents the entire population living in the
Kingdom, which is estimated at 34 million and 218 thousand people, and it represents the value of N. ${ }^{1}$ Given that the entire population is susceptible to the disease at the beginning of the pandemic, thus $S_{0}=N$, and the infection rate is $R_{0}=\frac{\beta}{\gamma}=4.3$. In Figure (9), a curve was fitted to estimate the cases of infection and the cumulative cases using the SIER model.

From figures 2 ,we notice that the SEIR model gives a good fitting of the timeline of the phenomenon, which is what has been confirmed by the value of the coefficient of determination $R^{2}=0.882$

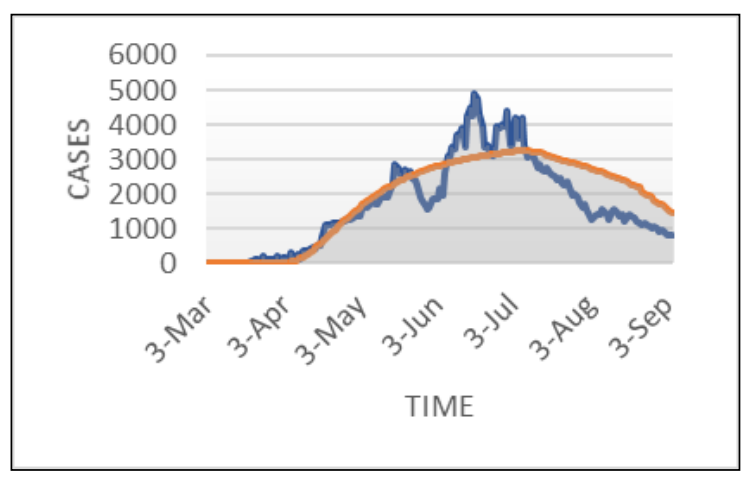

Figure 2: Daily prediction using SEIR model and actual values

\section{The results of regression models}

The results of estimating the proposed regression models can be summarized through Table (II), which shows the values of the regression coefficients, coefficient of determination, and their significance at $\alpha$ $=0.05$.

From Table (II) we observe the high value of $\boldsymbol{R}^{\mathbf{2}}$ for all polynomial models, as it ranged between 0.803 and 0.910 , and the best model for fitting the relationship was at $\mathrm{n}=4$.

Through figure (3), polynomial models give a good fit to the relationship curve more than the quadratic model. There is an improvement in the relationship fitting as $n$ increases until its value reaches 4 , then the model becomes insignificant after that. As a result, the best fitting for the relationship is via the polynomial model $(n=4)$. 
Table II: The parameter and coefficient of determination ${ }^{\left(\mathbf{R}^{2}\right)}$ for the regression models

\begin{tabular}{|c|c|c|c|c|c|c|}
\hline Models & $\beta_{a}$ & $\beta_{n}$ & $\beta_{x}$ & $\beta_{x}$ & $\beta_{4}$ & $R^{x}$ \\
\hline Quadratic & $\begin{array}{l}2.32 \\
(0.002)\end{array}$ & --- & $\begin{array}{l}0.032 \\
(0.00)\end{array}$ & --- & --- & $\begin{array}{l}0.065 \\
(0.00)\end{array}$ \\
\hline $\begin{array}{l}\text { Polynomial } \\
(n=2)\end{array}$ & $\begin{array}{l}1.03 \\
(0.00)\end{array}$ & $\begin{array}{l}78.271 \\
(0.00)\end{array}$ & $\begin{array}{l}0.365 \\
(0.00)\end{array}$ & --- & --- & $\begin{array}{l}0.803 \\
(0.00)\end{array}$ \\
\hline $\begin{array}{l}\text { Polynomial } \\
(n=3)\end{array}$ & $\begin{array}{l}3.86 \\
(0.00)\end{array}$ & $\begin{array}{l}17.961 \\
(0.00)\end{array}$ & $\begin{array}{l}0.003 \\
(0.00)\end{array}$ & $\begin{array}{l}0.448 \\
(0.00)\end{array}$ & --- & $\begin{array}{l}0.904 \\
(0.00)\end{array}$ \\
\hline $\begin{array}{l}\text { Polynomial } \\
(n=4)\end{array}$ & $\begin{array}{l}4.021 \\
(0.03)\end{array}$ & $\begin{array}{c}168.508 \\
(0.00)\end{array}$ & $\begin{array}{c}22.985 \\
(0.00)\end{array}$ & $\begin{array}{c}0.0021 \\
(0.00)\end{array}$ & $\begin{array}{c}2.85 \times 10^{-x} \\
(0.00)\end{array}$ & $\begin{array}{l}0.910 \\
(0.00)\end{array}$ \\
\hline
\end{tabular}

\section{DISCUSSION}

By analyzing the path of infection with the Covid-19 pandemic in Saudi Arabia, a decrease in infections has been noticed in recent days. Now, the important question is whether this is an indication of the disease receding or a new wave will appear in the coming days. Although the relationship fitting curves reflect different paths of these expectations, some of them tread the path of pessimism. It means that we should expect an increase in infections notwithstanding the recession of the pandemic wave happening right now as a series of chains of decline and rise in the degree of the spread of the pandemic.

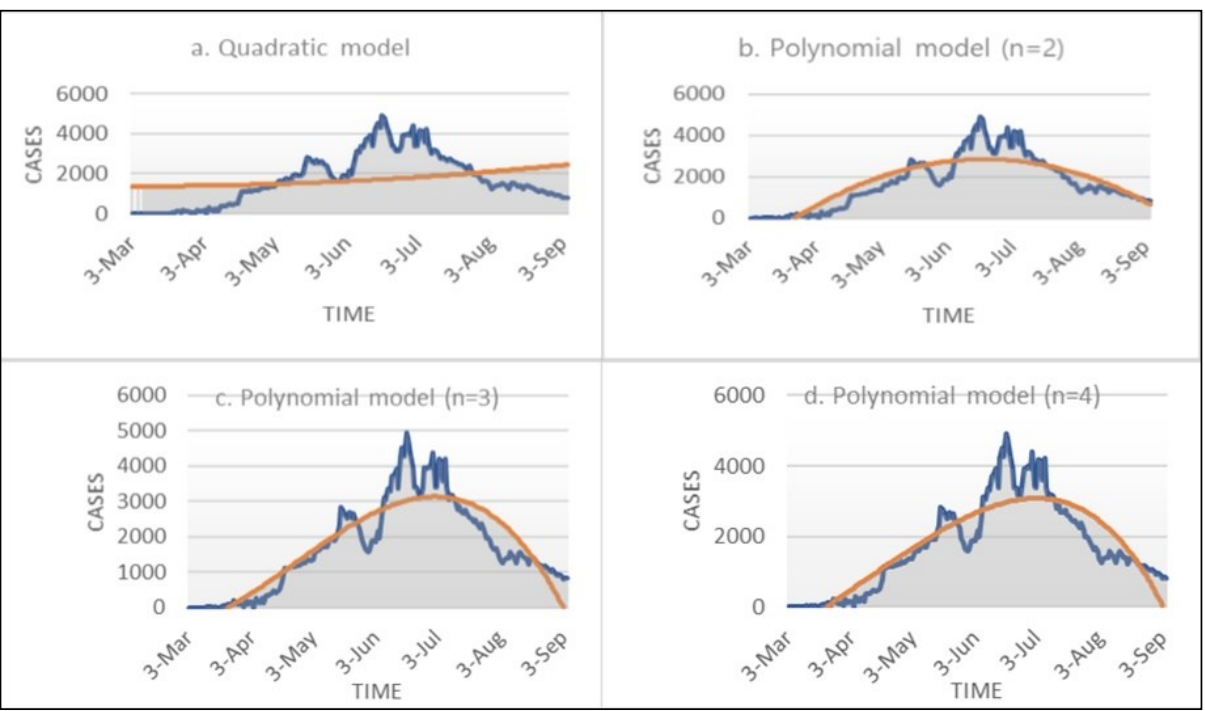

Figure 3: Daily prediction using quadratic and polynomial models and actual values

Gompertz, Mitscherlich and quadratic models show that there could be a rise in the short term while the SEIR model indicates that in the long term. Likewise, there are models that show the stability of infection rates such as the generalized logistic model. There are yet some other models that indicate recession of the pandemic, namely Weibull and Polynomial models. It's clear that the disappearance of disease in the long term will take more time according to the SEIR model, which is considered an effective model that works well for predicting epidemics, especially in the peak infection period. ${ }^{17}$ In addition, the SEIR model relies on the fact that there is no power to stop epidemics other than community acquisition of herd immunity. ${ }^{18}$ Henceforward, it can be said that in order to deal with the disease, a suitable and reliable vaccine must be available, which has begun to emerge now, where a set of vaccines have been distributed in many countries to limit the spread of the disease in the long run. This 
must be adopted that call for a return to normal life, while it is important to consider the necessary precautions, which prevent the spread of the disease by adopting the following steps:

1. Gradual re-opening of schools and universities, so that the educational process is in an organized manner through distance learning and student attendance.

2. Providing international aviation to some countries where the pandemic is heading to decline.

3. Allowing the performance of Umrah in an organized manner that takes into account the health requirements and decongestion.

Conversely, there must be alternative strategies for dealing with the spread of the pandemic so that it is contained quickly, and then conduct the appropriate lockdown. This may be done through the following steps:

1. A complete or partial lockdown and restriction of the movement of people in some or all cities.

2. Imposing restrictions on travel (exit and return).

3. Providing alternative field hospitals to deal with critical cases.

4. Resorting to distance education in schools and universities.

5. Imposing restrictions on the operation of restaurants and shops.

Owing to the need to keep commercial and economic movement floating, precautionary measures should not interfere with the progress of these activities in a flexible and productive manner. This can be done by adopting protocols to deal with economic and commercial activities in light of pandemics. Some countries have adopted a complete lockdown strategy. It is obvious that China's experience in dealing with SARS-CoV-2 virus through a complete lockdown, has been successful in controlling the Covid-19 virus. ${ }^{20}$ In fact, a fixed strategy should not be adopted all the time due to the economic and social problems that it entails, but different strategies must be used according to the infection status and the extent of the disease, which has proven successful in many countries. ${ }^{21}$

\section{CONCLUSION}

In this study, a set of mathematical and statistical models was used to fit the temporal curve of the number of cases of the Covid-19 pandemic in the Kingdom of Saudi Arabia, whether in the short or long terms using three sections of models. The first section is nonlinear growth models and it includes logistic model, generalized logistic model, Weibull model, Mitscherlich model, Gompertz model and the best model for fitting the relationship was the Weibull model. The second section includes the SEIR model, which can be used to fit the relationship in the long run, and it gave good results for the fitting of the relationship. The third section is the regression model and they include the quadratic and polynomial models at $\mathrm{n}=2,3,4$ and the best model for fitting the relationship in this section was the polynomial model at $n=4$. Through these models, it is possible to predict the direction of the disease in the future, as we note that there is a tendency for the disease to decline during the short period, with the expectation of other waves of the epidemic that will recede in the long term with the emergence of a suitable vaccine.

\section{ACKNOWLEDGEMENT}

We thank the Deanship of Scientific Research at Islamic University of Madinah for sponsoring this manuscript.

\section{REFERENCES}

1. $\mathrm{MOH}$, Saudi Arabia, [online]. Available at: (http// www.moh.gov.sa) Accessed September 10, 2020.

2. Huang C, Wang Y, Li X, et al. 2020. Clinical features of patients infected with 2019 novel coronavirus in Wuhan. China. Lancet (London, England), 395(10223):497-506. (https:// doi.org/10.1016/S0140-6736(20)30183-5)

3. Malavikaa B., Marimuthua S., Melvin J., Ambily N., Edwin S. 2020. Jeyaseelanc I. Forecasting COVID -19 epidemic in India and high incidence states using SIR and logistic growth models. Clinical Epidemiology and Global Health.9 :26-33. (https://doi.org/10.1016/j.cegh.2020.06.006) 
4. Ahmad S., Fadi A., Negar M., Seyed A. 2020. Prediction of COVID-19 Dynamics in Kuwait using SIRD Model. Integrative Journal of Medical Sciences. 7, ID 170.

5. Gaurav P., Poonam C., Rajan G., Saibal P. 2020. SEIR and Regression Model based COVID-19 outbreak predictions in India. ArXiv preprint arXiv: 2004.00958, arxiv.org.

6. Christine S., John W., Kathy K. et al.2020. How simulation modelling can help reduce the impact of COVID-19. Journal of Simulation. 14(2).

DOI: $10.1080 / 17477778.2020 .1751570$

7. Seleh I., Ibrahim A., Ebrahim A. 2020. Forcasting the spreat of the COVID-19 pandemic in Saudia using ARIMA prediction model under current public health interventions. Journal of infection and public health. 13:914-919.

8. Villalobos A., Mario A. 2020. Using generalized logistics regression to forecast population infected by Covid-19.: arXiv: 2004.02406v1 [q-bio.PE].

9. Richards, F. J. 1959.A Flexible Growth Function for Empirical Use". Journal of Experimental Botany. 10 (2): 290-300. Doi:10.1093/jxb/10.2.290.

10. Fréchet, M. 1927.Sur la loi de probabilité de l'écart maximum. Annales de la Société Polonaise de Mathematique, Cracovie. 6: 93-116.

11. Kermack W., McKendrick A. 1991.Contributions to the mathematical theory of epidemics. Bulletin of Mathematical Biology. 53 (1-2): 33-55.

12. Yang C., Wang J. 2020. A mathematical model for the novel coronavirus epidemic in Wuhan- Chins. Mathematical Biosciences and Engineering. 17(3).

13. Aron J. 1984. Seasonality and Period-doubling Bifurcations in an Epidemic Model", Journal of Theoretical Biology. 110(4).

14. Worldometers, Saudi Arabia. [online]. Available at: (https://www.worldometers.info/coronavirus/) Accessed september 10, 2020.

15. Xia, W., Liao, J., Li, C., et al. 2019. Transmission of corona virus disease 2019 during the incubation period may lead to a quarantine loophole MedRxiv, 020.03.06.20031955. (https:// doi.org/10.1101/2020.03.06.20031955)

16. Abdullah A., Naif K., Mazen H., Anwar M. 2020. Preparedness and response to COVID-19 in Saudi Arabia: Building on MERS experience. Journal of Infection and Public Health. 13, (6): 834-838.
17. Ahmad N. 2020. COVID-19 Modeling in Saudi Arabia Using the Modified Susceptible-ExposedInfectious-Recovered (SEIR) Model. Cureus. 12 (9):e10452. Doi:10.7759/cureus.10452

18. Andrew G.2020. On Using SIR Models to Model Disease Scenarios for COVID-19. Quarterly Review.14 (1).

19. Uzma F. 2016. Middle East respiratory syndrome coronavirus (MERS-CoV): Impact on Saudi Arabia, 2015. Saudi Journal of Biological Sciences. 25: 1402-1405.

20. Muhammad A., Hafiz U., Khan N., Abeer K., Kainat S, Muhammad S. 2020. COVID-19 around the world and the Chinese strategy to cope with SARS-CoV-2The Vietnamese Journal of Biomedicine. 7(7): 3890-3897.

21. Jon P., 2020. Nudges against pandemics: Sweden's COVID-19 containment strategy in perspective. Policy and society. 39(3): 478-493. 